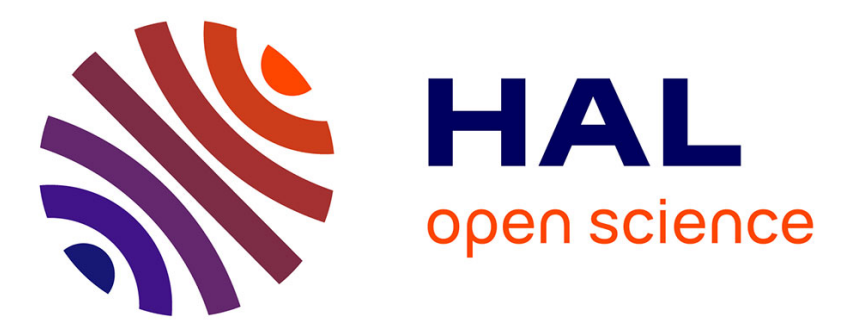

\title{
Corrélation entre la liaison chimique et la structure des états vitreux et leurs propriétés thermodynamiques et cinétiques
}

D. Weigel

\section{- To cite this version:}

D. Weigel. Corrélation entre la liaison chimique et la structure des états vitreux et leurs propriétés thermodynamiques et cinétiques. Revue de Physique Appliquée, 1977, 12 (5), pp.637-645. 10.1051/rphysap:01977001205063700 . jpa-00244219

\section{HAL Id: jpa-00244219 https://hal.science/jpa-00244219}

Submitted on 1 Jan 1977

HAL is a multi-disciplinary open access archive for the deposit and dissemination of scientific research documents, whether they are published or not. The documents may come from teaching and research institutions in France or abroad, or from public or private research centers.
L'archive ouverte pluridisciplinaire HAL, est destinée au dépôt et à la diffusion de documents scientifiques de niveau recherche, publiés ou non, émanant des établissements d'enseignement et de recherche français ou étrangers, des laboratoires publics ou privés. 


\title{
CORRÉLATION ENTRE LA LIAISON CHIMIQUE ET LA STRUCTURE DES ÉTATS VITREUX ET LEURS PROPRIÉTÉS THERMODYNAMIQUES ET CINÉTIQUES
}

\author{
D. WEIGEL
}

Laboratoire de Chimie-Physique du Solide (*), Ecole Centrale des Arts et Manufactures, 92290 Chatenay-Malabry, France

\begin{abstract}
Résumé. - Nous montrons que la rigidité des liaisons chimiques et la structure des solides non cristallins conditionnent leurs propriétés thermodynamiques et cinétiques, d'une part, et leur module d'élasticité en traction en dessous de la transition vitreuse d'autre part ; nous appliquons rapidement ces notions à la mise en forme des matériaux correspondants.
\end{abstract}

Abstract. - We show the chemical bond's stiffness explain the thermodynamic and cinetic properties of non cristalline solids and also their Young modulus below the glassy transition; we shortly applied these ideas to form non cristallin materials.

Deux facteurs principaux conditionnent les propriétés de changement d'état des solides non cristallins :

- La rigidité des liaisons chimiques qui assurent leur cohésion, cf. paragraphe 1 .

- L'agencement structural de ces liaisons.

Ces deux facteurs expliquent aussi la rigidité macroscopique en traction du solide élastique caractérisée par son module d'Young aux températures inférieures à $T_{\mathrm{g}}$, cf. paragraphe 2 .

Au paragraphe 3 nous présentons le changement progressif d'état d'un verre en distinguant la transition vitreuse $\left(T_{\mathrm{g}}\right)$ des transitions thermodynamiques.

L'intervalle de température où la viscosité du solide non cristallin décroît rapidement lors du changement continu d'état dépend principalement des deux facteurs étudiés au début de cet exposé, cf. paragraphe 4.

Enfin nous présentons rapidement le phénomène de dévitrification nuisible aux qualités d'un verre, mais qu'on provoque volontairement lors de la mise en forme d'un verre céramique.

1. Rigidité des liaisons covalentes et ioniques. L'énergie des liaisons ioniques est aussi importante que celles des liaisons covalentes et on a coutume de dire qu'il s'agit de deux liaisons fortes.

$$
\begin{aligned}
& \mathrm{H}-\mathrm{Cl}: 104 \mathrm{kcal} / \mathrm{mole}, \\
& \mathrm{Na}^{+}-\mathrm{Cl}^{-}: 185 \mathrm{kcal} / \mathrm{mole} ; \\
& \mathrm{Ca}^{++}-\mathrm{O}^{--}: 825 \mathrm{kcal} / \mathrm{mole},
\end{aligned}
$$

Ces deux valeurs correspondent aux énergies réticulaires des cristaux $\mathrm{NaCl}$ et $\mathrm{CaO}$.

Mais la liaison ionique est beaucoup moins rigide que la liaison covalente comme le prouve les valeurs

(*) E. R. A. au C. N. R. S. $n^{\circ} 456$. des constantes de force obtenues à partir d'expériences de spectroscopie sur les gaz $(\mathrm{HCl})$ ou sur les solides $(\mathrm{NaCl}$ et $\mathrm{CaO})[1]$.

$$
\begin{aligned}
& \mathrm{H}-\mathrm{Cl} k=5 \times 10^{5} \text { dyn./cm } \\
& \mathrm{NaCl} k=0,11 \times 10^{5} \text { dyn. } / \mathrm{cm}
\end{aligned}
$$

et

$$
\mathrm{CaO} \quad k=0,27 \times 10^{5} \mathrm{dyn} . / \mathrm{cm} .
$$

On peut ainsi schématiser sur les deux graphes $U_{\mathrm{e}}(r)$ les caractéristiques énergétiques et mécaniques de ces deux types de liaison ; la courbure au minimum du graphe est fixée par la valeur de $k$ car l'équation de la parabole osculatrice est $U_{\mathrm{e}}=\frac{1}{2} k\left(r-r_{0}\right)^{2}$, dans l'approximation de l'oscillateur harmonique : (Fig. 1).
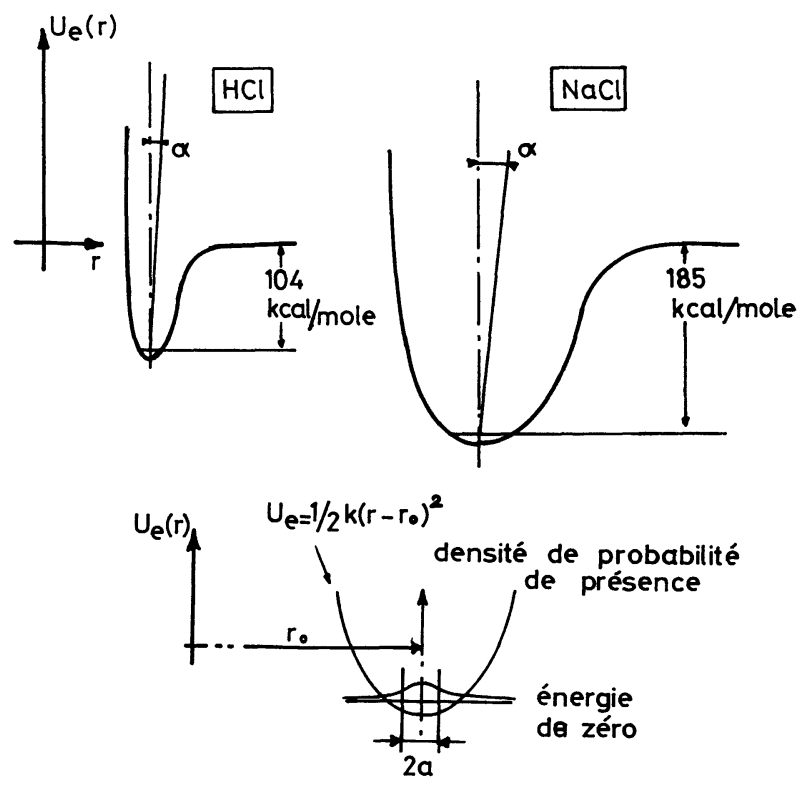

FIG. 1. - Graphe $U_{\mathrm{e}}(r)$ pour $\mathrm{HCl}$ gazeux et $\mathrm{NaCl}$ cristal : $U_{\mathrm{e}}=$ énergie électronique de la molécule ou du cristal $; r=$ distance entre deux noyaux plus proches voisins ; $a=$ amplitude moyenne de vibration à $0 \mathrm{~K}$. 
TABLEAU I

\begin{tabular}{|c|c|c|c|c|}
\hline & $\begin{array}{c}\text { Rigidité } \\
C_{11} \times 10^{12} \text { dynes } / \mathrm{cm}^{2} \\
\quad[\end{array}$ & Fusion, $\mathrm{K}$ & $\begin{array}{c}\begin{array}{c}\text { Dilatation } \\
\text { thermique }\end{array} \\
\frac{\Delta l}{l \Delta T} \times 10^{-6} \mathrm{~K}^{-1}\end{array}$ & $\begin{array}{l}\text { Dureté } \\
\text { échelle } \\
\text { de MOH }\end{array}$ \\
\hline C diam. & 10,75 & $\sim 4000$ & 1,2 & 10 \\
\hline $\mathrm{Al}_{2} \mathrm{O}_{3}$ & 4,7 & 2325 & $\sim 9$ & 9 \\
\hline $\mathrm{SiO}_{2}$ cristobalite & 0,78 & 1993 & $\sim 9$ & 7 \\
\hline $\mathrm{NaCl}$ & 0,49 & 1050 & 40 & 2 \\
\hline K & 0,037 & 336 & 83 & 0,5 \\
\hline
\end{tabular}

On peut donc prévoir $\left({ }^{1}\right)$ que dans un solide covalent les vibrations des noyaux ont de petites amplitudes et de grandes fréquences alors qu'elles ont de grandes amplitudes et de faibles fréquences dans un solide ionique. Les amplitudes moyennes de vibration, $a$, à zéro $\mathrm{K}$, déterminées expérimentalement par la diffraction des RX ou des neutrons confirment parfaitement cette prévision :

$-0,045 \AA$ pour le carbone diamant

- $0,125 \AA$ pour le chlorure de sodium

$-0,28 \AA$ A pour le sodium métallique où la liaison est encore beaucoup moins rigide que la liaison ionique (Fig. 1 et tableau I).

Maintenant il est logique de penser qu'une grande rigidité, donc de faibles amplitudes de vibration, implique un caractère réfractaire marqué ainsi qu'une faible dilatation thermique et une grande dureté.

C'est ce que nous confirme le tableau I qui établit la même relation d'ordre sur ces quatre propriétés physiques pour 5 solides types.

En effet le module d'élasticité $C_{11}$ caractérise bien la rigidité du cristal directement induite par la rigidité des liaisons qui le constituent (et leur agencement structural); de faibles amplitudes de vibration favorisent évidemment une température (et une enthalpie molaire) de fusion élevée ; pour une même anharmonicité une liaison souple conduit à un grand coefficient $\alpha$ de dilatation linéaire contrairement à une liaison rigide, ainsi qu'on le constate sur les graphes $U_{\mathrm{e}}(r)$ de la figure 1 où la droite inclinée est le lieu géométrique des milieux des cordes horizontales; enfin une grande rigidité de liaison ne favorise pas l'écartement des atomes lors de l'enfoncement d'un corps étranger en surface au cours d'une mesure de dureté.

On peut ainsi classer les différents types de liaisons chimiques par rigidité croissante.

Liaison de Van der Waals, liaison métallique $(\mathrm{K})$, liaison ionique 1-1 $(\mathrm{NaCl})$, liaison ionique 2-2 $(\mathrm{CaO})$ et liaison ionique $50 \%$ covalente $50 \%\left(\mathrm{SiO}_{2}\right)$, liaison métallique avec covalence partielle $(\mathrm{Fe}, \mathrm{Ni})$, liaison

(1) On rappelle que pour le vibrateur harmonique la fréquence de vibration $v_{0}$ vaut $v_{0}=\frac{1}{2 \pi} \sqrt{\frac{k}{m}}$, où $m$ est la masse réduite. ionique 2-3 avec covalence partielle marquée $\left(\mathrm{Al}_{2} \mathrm{O}_{3}\right)$ liaison covalente (C diamant).

On sait, en effet, que la différence d'électronégativité de Pauling entre les deux partenaires d'une liaison permet d'en prévoir approximativement le pourcentage de caractère ionique ; ainsi on obtient les pourcentages ioniques suivants pour les principales liaisons des verres d'oxydes :

$$
\begin{gathered}
\mathrm{B}_{2} \mathrm{O}_{3}: 43 \% ; \quad \mathrm{SiO}_{2}: 50 \% ; \\
\mathrm{AL}_{2} \mathrm{O}_{3}: 63 \% ; \quad \mathrm{CaO}: 80 \% ; \quad \mathrm{Na}_{2} \mathrm{O}: 83 \% .
\end{gathered}
$$

2. Verres d'oxydes, solides iono-covalents. - Considérons maintenant le deuxième facteur qui conditionne les propriétés physiques et thermodynamiques des solides c'est-à-dire l'agencement des atomes et en particulier la coordinance du cation.

Une des conditions $\left({ }^{2}\right)$ énoncées par Zachariasen pour obtenir un verre d'oxyde est que la coordinance du cation soit faible ( 3 ou 4 ) ; en effet il faut laisser au désordre partiel la place de s'établir.

Etudions ainsi les deux verres d'oxydes suivants :

a) Dans la silice vitreuse, $\mathrm{SiO}_{2}$, le silicium, formateur du réseau tridimensionnel désordonné a la coordinance tétraédrique 4 et deux tétraèdres voisins sont liés entre eux par un seul atome d'oxygène. Les distances $\mathrm{Si}-\mathrm{O}$ et O-O valent 1,62 et $2,65 \AA \AA$ et la valeur maximum de la distribution des angles $\widehat{\mathrm{SiOSi}}$ est de $144^{\circ}$ [2]. Cette structure est proche de celle du cristal cubique de cristobalite $\left({ }^{3}\right) \beta$, variété de la silice cristalline en équilibre thermodynamique avec le liquide à $1710^{\circ} \mathrm{C}$.

Sachant que le verre plat, le verre creux et la glace sont constitués principalement de \#70\% de $\mathrm{SiO}_{2}$, de $14 \%$ de $\mathrm{Na}_{2} \mathrm{O}$ et de 8 à $11 \%$ de $\mathrm{CaO}$, étudions les effets de l'addition des perturbateurs $\mathrm{Na}_{2} \mathrm{O}$ puis $\mathrm{CaO}$ dans le réseau désordonné de la silice vitreuse.

Le graphe de la figure $2 c$ nous montre que l'addition des oxydes d'alcalins $\mathrm{Na}_{2} \mathrm{O}$ et $\mathrm{K}_{2} \mathrm{O}$ provoque une perte

(2) Les autres conditions sont les suivantes: Un oxygène ne doit pas être lié à plus de 2 cations et les polyèdres de coordination des cations voisins ne doivent avoir que des coins oxygènes en commun, ...

(3) Il y a un léger désordre dans ce cristal, les atomes d'oxygène se trouvant répartis sur des cercles de rayon $0,4 \AA$ dont l'axe est la droite joignant deux siliciums plus proches voisins. 


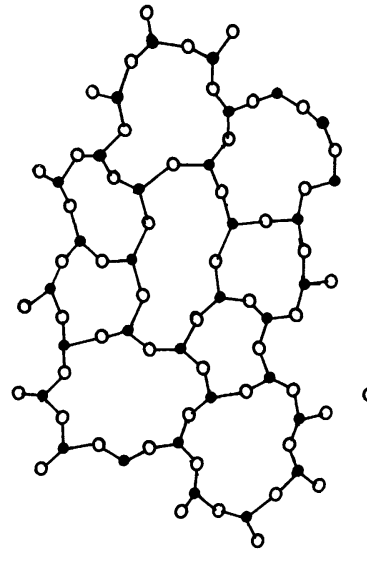

- $\mathrm{Si}$

(a)

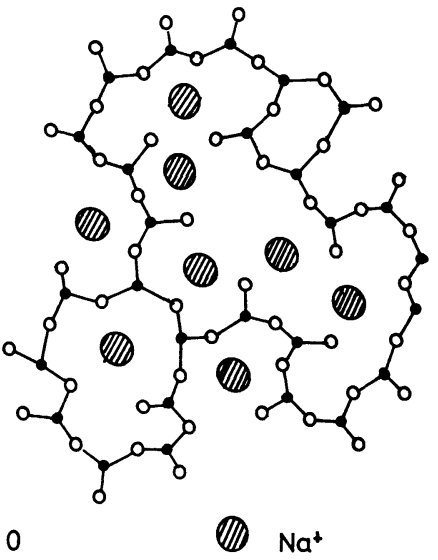

(b)

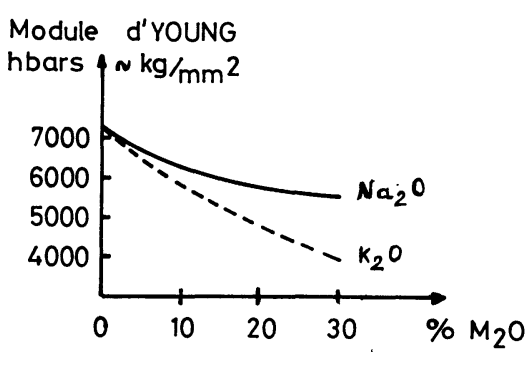

(c)

Fig. 2. - a) Représentation à deux dimensions du réseau tridimensionnel de la silice vitreuse. $b$ ) Même représentation d'un verre doux au sodium. $c$ ) Effet de l'addition de $\mathrm{Na}_{2} \mathrm{O}$ et $\mathrm{K}_{2} \mathrm{O}$ sur le module d'Young de la silice vitreuse.

notable de la rigidité du solide au cours d'une expérience de traction [3].

En effet la liaison ionique $\mathrm{Na}-\mathrm{O}$ étant beaucoup plus souple que la liaison covalente $\mathrm{Si}-\mathrm{O}$ on peut donc schématiser un verre doux au sodium sur la figure $2 b$.

Les ions $\mathrm{Na}^{+}$se trouvent dans des cavités où ils se coordonnent avec environ six oxygènes non pontants $\mathrm{O}^{\delta-}$ qui ne sont plus liés qu'à un seul Si par une liaison rigide $\mathrm{Si}-\mathrm{O}$ contrairement aux oxygènes pontants qui sont liés à deux atomes de silicium voisins par des liaisons rigides.

Naturellement cet effet est accentué avec $\mathrm{K}_{2} \mathrm{O}$ puisque la liaison $\mathrm{K}-\mathrm{O}$ est encore plus ionique et que l'ion $\mathrm{K}^{+}$est plus gros que l'ion $\mathrm{Na}^{+}$.

Cette perte de rigidité par addition d'oxyde perturbateur se retrouve sur le diagramme binaire de solidification $\mathrm{SiO}_{2}-\mathrm{Na}_{2} \mathrm{O}$ de la figure $3 a$.

En effet l'addition d'environ $25 \%$ de $\mathrm{Na}_{2} \mathrm{O}$ dans la silice vitreuse permet d'avoir le liquide en équilibre thermodynamique avec l'eutectique à une température aussi basse $\left({ }^{4}\right)$ que $780{ }^{\circ} \mathrm{C}$, au lieu de $1710^{\circ} \mathrm{C}$ pour $\mathrm{SiO}_{2}$ pur.

(4) Dans le diagramme de solidification $\mathrm{SiO}_{2}-\mathrm{K}_{2} \mathrm{O}$, l'eutectique correspond à la température de $720^{\circ} \mathrm{C}$, légèrement inférieure à $780^{\circ} \mathrm{C}$

REVUE DE PHYSIQUE APPLIQUÉE. - T. 12, No 5, MAI 1977

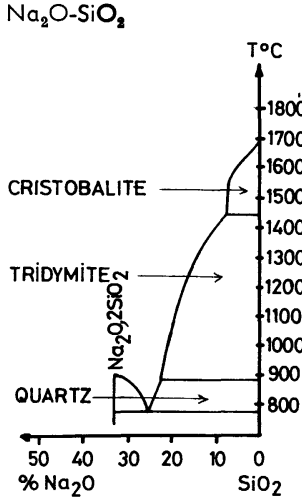

(a)

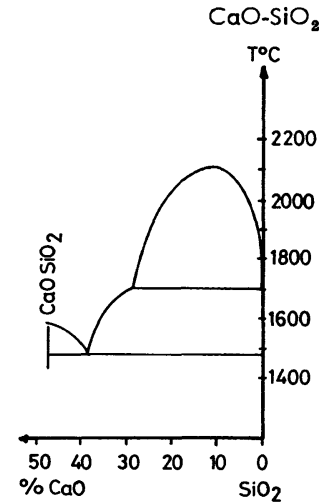

(b)
FIG. 3. - a) Diagramme de solidification $\mathrm{SiO}_{2}-\mathrm{Na}_{2} \mathrm{O}$. b) Diagramme de solidification $\mathrm{SiO}_{2}-\mathrm{CaO}$.

L'addition du troisième constituant, environ $10 \%$ de $\mathrm{CaO}$, conduit à la restauration du module d'Young soit 7200 hbars, valeur commune à la silice vitreuse et au verre silico-sodocalcique. On a donc compensé l'assouplissement du matériau dû à l'addition de $\mathrm{Na}_{2} \mathrm{O}$ par la liaison ionique $2-2, \mathrm{Ca}^{2+}-\mathrm{O}^{2-}$, qui est un peu plus rigide que la liaison $\mathrm{Si}-\mathrm{O}\left({ }^{5}\right)$.

On vérifie d'ailleurs sur le diagramme binaire de solidification $\mathrm{SiO}_{2}-\mathrm{CaO}$ qu'on doit ajouter $37 \%$ de $\mathrm{CaO}$ dans $\mathrm{SiO}_{2}$ pour n'abaisser la température de l'eutectique que de $230^{\circ} \mathrm{C}\left(1480^{\circ} \mathrm{C}\right.$ au lieu de $\left.1710^{\circ} \mathrm{C}\right)$ par rapport à la fusion de la cristobalite.

b) Dans le verre d'oxyde de bore, $\mathrm{B}_{2} \mathrm{O}_{3}$, le bore, formateur du réseau tridimensionnel désordonné à la coordinance triangulaire 3 et les groupements boroxyls hexagonaux sont liés entre eux par des oxygènes pontants avec quelques groupes $\mathrm{BO}_{3}$ qui ne forment pas d'anneaux $\left({ }^{6}\right)$ (Fig. 4a).

Cette coordinance très faible correspond à une réticulation assez lâche qui conduit à un faible module d'Young pour ce verre : 1800 hbars.

L'expérience nous montre que l'addition du perturbateur $\mathrm{Na}_{2} \mathrm{O}$ implique une augmentation importante de ce module d'Young cf. figure $4 b$. Dans un tel verre de borate de sodium l'addition de $\mathrm{Na}_{2} \mathrm{O}$ provoque un changement de coordination du bore de 3 $(\mathrm{B}-\mathrm{O}=1,37 \AA)$, à $4(\mathrm{~B}-\mathrm{O}=1,48 \AA)$, les ions $\mathrm{Na}^{+}$se plaçant au voisinage des tétraèdres $\mathrm{BO}_{4}^{-}$sans rompre

(5) D'après les valeurs de $k$ la liaison Ca-O est 2,5 fois plus rigide que la liaison $\mathrm{Na}-\mathrm{Cl}$ alors que les valeurs de $C_{11}$ nous montrent que la liaison $\mathrm{Si}-\mathrm{O}$ est 1,5 fois plus rigide, que la liaison $\mathrm{NaCl}$.

D'ailleurs lorsqu'on cherche à augmenter la rigidité macroscopique en traction d'un verre on diminue le pourcentage de silice et on augmente celui de $\mathrm{CaO}$; exemple d'un verre de module d'Young de 12000 hbars (contre 7200 pour celui de la silice vitreuse) $: \mathrm{SiO}_{2}(43 \%) \mathrm{CaO}(19 \%) \mathrm{MgO}(19 \%) \mathrm{Al}_{2} \mathrm{O}_{3}$ (11\%) $\mathrm{ZrO}_{2}(5,5 \%) \ldots$

(6) La distance $\mathrm{B}-\mathrm{O}$ est alors de $1,37 \AA$ et les angles $\widehat{\mathrm{BOB}}$ de 130 degrés environ. 


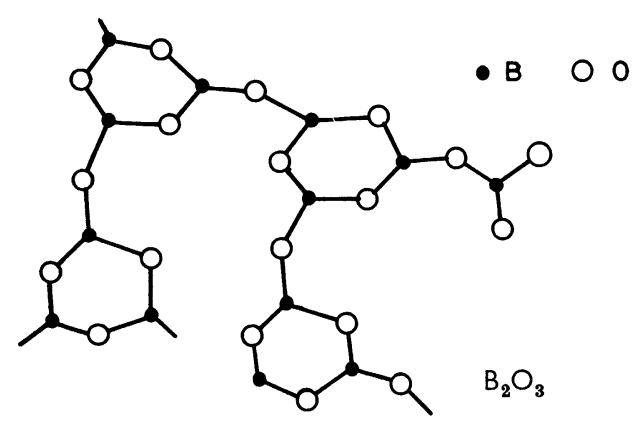

(a)

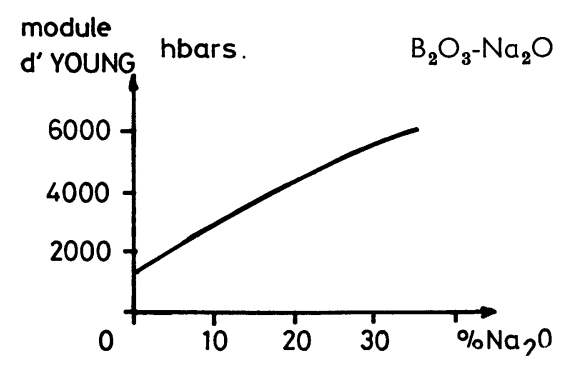

(b)

Fig. 4. - a) Représentation à deux dimensions du réseau tridimensionnel de l'oxyde de bore vitreux. $b$ ) Effet de l'addition de $\mathrm{Na}_{2} \mathrm{O}$ sur le module d'Young de $\mathrm{B}_{2} \mathrm{O}_{3}$.

le réseau désordonné du formateur qui est suffisamment lâche pour les accepter [5, 6].

On s'explique ainsi l'augmentation des valeurs du module d'Young et de la température de transition vitreuse provoquée par l'addition d'oxyde de sodium.

Nous avons donc vu que dans le réseau du formateur tétracoordonné $\mathrm{SiO}_{2}, \mathrm{Na}_{2} \mathrm{O}$ est un perturbateur adoucisseur alors qu'il se comporte comme un perturbateur durcisseur dans le réseau du formateur tricoordonné $\mathrm{B}_{2} \mathrm{O}_{3}$. De même dans le verre pyrex on adoucit le réseau désordonné du formateur $\mathrm{SiO}_{2}$ en le perturbant par le formateur $\mathrm{B}_{2} \mathrm{O}_{3}$ à réticulation beaucoup plus lâche.

3. Les transitions thermodynamiques et la transition vitreuse. - Lorsqu'on réchauffe de la cristobalite $\beta\left(\mathrm{SiO}_{2}\right)$ on observe sur le graphe volume spécifique fonction de la température une discontinuité à $1710^{\circ} \mathrm{C}$ qui correspond à la fusion de la cristobalite, (Fig. 5a).

Il s'agit d'une transition du $1^{\text {er }}$ ordre au sens d'Ehrenfest puisque le volume est une dérivée première de l'enthalpie libre : $v=(\partial G / \partial p)_{s}$.

Lorsqu'on refroidit cette silice fondue, on obtient un liquide surfondu de plus en plus visqueux, puis, vers $1100^{\circ} \mathrm{C}$, on observe un changement de pente sur le graphe $V(T)$, la droite en dessous de cette température étant presque parallèle à la droite caractéristique du réchauffage de la silice cristallisée.

La température du changement de pente correspond à la transition vitreuse, qu'on note $T_{\mathrm{g}}$, puisque le solide rigide observé pour $T<T_{\mathrm{g}}$ est non cristallin :

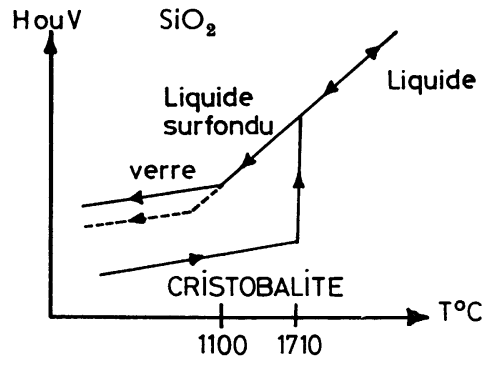

(a)

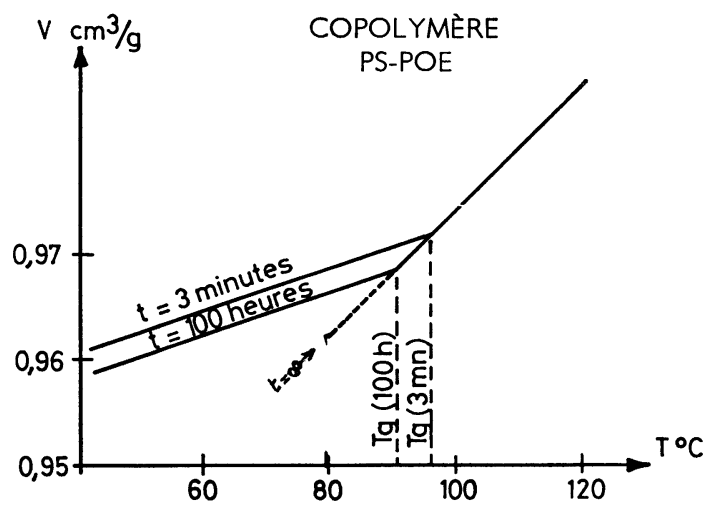

(b)

FIG. 5. - a) Chauffage et refroidissement de la silice. $b$ ) Trempe intermédiaire, $115 \mathrm{C} \rightarrow T$, d'un copolymère $\mathrm{PS}-\mathrm{POE}$.

On pourrait penser qu'il s'agit d'une transition thermodynamique du $2^{\mathrm{e}}$ ordre au sens d'Ehrenfest, qui s'accompagne de discontinuités sur les grandeurs reliées aux dérivées secondes de l'énergie libre : le coefficient de dilatation volumique

$$
\alpha_{\mathrm{v}}=\frac{1}{V} \frac{\partial^{2} G}{\partial p \partial T}
$$

ou la chaleur spécifique à pression constante :

$$
C_{\mathrm{p}}=-\frac{T \partial^{2} G}{\partial T^{2}}
$$

et qui correspondent donc à des points anguleux sur les graphes $V(T)$ ou $H(T)$, comme sur la figure $5 a$.

Mais on remarque d'abord que la température de transition vitreuse, $T_{g}$, dépend beaucoup de la cinétique. On opère une trempe intermédiaire sur un copolymère vitreux polystyrène $(\mathrm{PS})$ polyoxyéthylène (POE) depuis la température $115^{\circ} \mathrm{C}$ jusqu'à la température $T$ et on a porté sur le graphe de la figure $5 b$ le graphe du volume spécifique mesuré $3 \mathrm{~min}$. et $100 \mathrm{~h}$ après la trempe. On a, d'autre part, évalué le volume spécifique au temps infini en utilisant le principe de superposition température-temps et le graphe correspondant $V(T)_{t=\infty}$ est dans le prolongement de la couche d'équilibre du liquide surfondu. Ainsi aux températures inférieures à $T_{\mathrm{g}}$ le volume diminue lentement jusqu'à atteindre (théoriquement après un temps qui peut être très long) la valeur $V(T)_{t=\infty}$ et ce phénomène est appelé stabilisation de l'état vitreux. 
La température de transition vitreuse, $T_{\mathrm{g}}$ ne peut donc être définie avec précision qu'à l'aide d'une norme précisant :

- la propriété physique utilisée,

- l'histoire thermique antérieure de l'échantillon,

- les conditions expérimentales (vitesse de refroidissement, ...)

La valeur de $T_{\mathrm{g}}$ dépend donc sensiblement de la norme choisie.

Ensuite l'entropie au zéro Kelvin n'est pas nulle pour les états vitreux (elle vaut $0,9 \mathrm{cal} / \mathrm{mole} \mathrm{K}$ pour la silice vitreuse) le verre reste donc désordonné jusqu'au $0 \mathrm{~K}$ et on peut dire qu'en dessous de $T_{\mathrm{g}}$ le verre n'est pas en état d'équilibre thermodynamique stable. La transition vitreuse n'est pas une transition thermodynamique : c'est un processus de figeage ; on peut dire que le verre est un liquide surfondu figé.

Précisons maintenant le processus cinétique de formation d'un verre.

Pour qu'un liquide cristallise au refroidissement il faut non seulement que la vitesse d'apparition des germes cristallins, c'est-à-dire la vitesse de nucléation, soit suffisante mais encore que la vitesse de croissance du cristal soit assez élevée dans un même intervalle de température.

On a tracé les graphes représentant ces deux vitesses en fonction de la température sur la figure 6 dans deux cas typiques. (a)

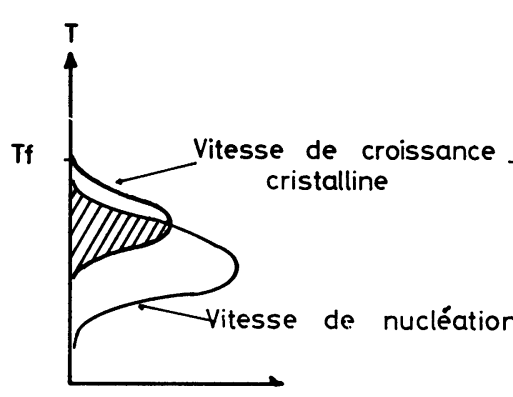

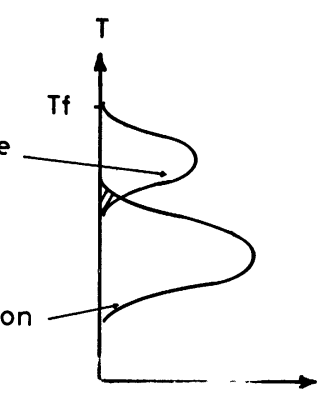

(b)
FIG. 6. $-a)$ Graphes vitesses $=f(T)$ dans le cas de la cristallisation. $b$ ) Graphes vitesses $=f(T)$ dans le cas de la formation d'un verre par refroidissement.

Sur la figure $6 a\left({ }^{7}\right)$ il y a recouvrement entre les deux graphes dans la zone hachurée et le solide cristallise au refroidissement ; au contraire, sur la figure $6 b\left({ }^{7}\right)$, la

(7) La vitesse de nucléation est proportionnelle au produit

$$
\exp \left(-\frac{W^{*}}{R T}\right) \exp \left(-\frac{\Delta G^{\prime}}{R T}\right)
$$

où $W^{*}$ est la barrière thermodynamique de nucléation et $\Delta G^{\prime}$ la barrière cinétique de nucléation.

Le premier facteur est faible pour les températures élevées et croît lorsque la température s'abaisse alors que le comportement du second facteur est naturellement opposé ce qui conduit à la forme du graphe vitesse de nucléation $=f(T)$.

Quant à la vitesse de croissance cristalline elle augmente avec la température, puis diminue évidemment lorsqu'on se rapproche de la température de fusion, $T_{\mathrm{f}}$. zone de recouvrement est négligeable et on observe la formation d'un liquide surfondu qui se fige ensuite à l'état de verre.

Pour les corps simples $\mathrm{SiO}_{2}, \mathrm{GeO}_{2}, \mathrm{~B}_{2} \mathrm{O}_{3}$ les énergies d'activation contrôlant la cristallisation sont du même ordre de grandeur que l'énergie d'activation de la viscosité, c'est-à-dire que la croissance cristalline est faible si la viscosité du liquide est grande.

Or on trouve dans le tableau II les valeurs des viscosités de 6 liquides à leur température de fusion cristalline, $T_{\mathrm{f}}$.

$\begin{array}{lcc} & \begin{array}{c}\text { TABleaU II } \\ \text { Température } \\ \text { de fusion, } \\ T_{\mathrm{f}} \text {, degrés } \mathrm{C}\end{array} & \begin{array}{c}\text { Viscosité } \\ \text { poises }\end{array} \\ \overline{\mathrm{LiCl}} & - & - \\ \mathrm{Fe} & 613 & 2 \times 10^{-2} \\ \mathrm{BeF}_{2} & 1535 & 7 \times 10^{-2} \\ \mathrm{~B}_{2} \mathrm{O}_{3} & 510 & 10^{5} \\ \mathrm{GeO}_{2} & 450 & 10^{5} \\ \mathrm{SiO}_{2} & 1115 & 10^{7} \\ \end{array}$

On vérifie naturellement que le liquide ionique $\mathrm{LiCl}$ est très peu visqueux puisque la liaison est souple, de même que le liquide métallique $\mathrm{Fe}$, malgré la covalence partielle de la liaison.

Par contre les liquides iono-covalents à liaisons plus rigides sont très visqueux, même à températures élevées : $10^{7}$ poises pour $\mathrm{SiO}_{2}$ et $\mathrm{GeO}_{2}$ où la réticulation est forte à cause de la coordinance 4 du cation et $10^{5}$ poises seulement pour $\mathrm{B}_{2} \mathrm{O}_{3}$ où la réticulation est beaucoup plus lâche à cause de la coordinance $3 \mathrm{du}$ bore.

$\mathrm{BeF}_{2}$ constitue une exception pour les liquides ioniques, sa grande viscosité conduisant à la formation d'un verre puisqu'elle gène la cristallisation.

4. Liaison, structure et échelle de température de la transition vitreuse des verres et plastiques. Viscosité en fonction de la température. - A la température ambiante un verre minéral ou organique est rigide et assez fragile : sa viscosité est très élevée $10^{19}$ poises environ pour le verre silico-sodocalcique.

La viscosité diminue lorsque la température augmente suivant le graphe caractéristique de la figure $7 a$ où l'état vitreux se comporte successivement comme :

- un solide élastique,

- puis un solide visco-élastique,

- puis un liquide visqueux.

Deux températures définies arbitrairement correspondent approximativement à ces changements qualitatifs d'états.

- La température de transition vitreuse, $T_{\mathrm{g}}$, définie au paragraphe précédent à l'aide de propriétés physiques telles que : le volume spécifique ou la dilatation thermique. 
Lorsqu'on définit $T_{\mathrm{g}}$ à l'aide de la viscosité, il s'agit de la température pour laquelle la viscosité du verre est de $10^{13,3}$ poises et cette température est légèrement supérieure à la température de contrainte $\left(10^{14,5}\right.$ poises) où la relaxation des contraintes résiduelles devient rapide.

- La température de ramolissement, $T_{\mathrm{L}}$, ou point de Littelton correspond, par définition, à la viscosité $10^{7,6}$ poises ; elle situe bien la zone de température où un verre peut être soufflé, lorsque sa viscosité vaut de $10^{6}$ à $10^{9}$ poises.

Comparons maintenant les graphes $\log \eta=f(T)$ pour les quatre verres de la figure $7 b$ dont la composition chimique est donnée dans le tableau III.

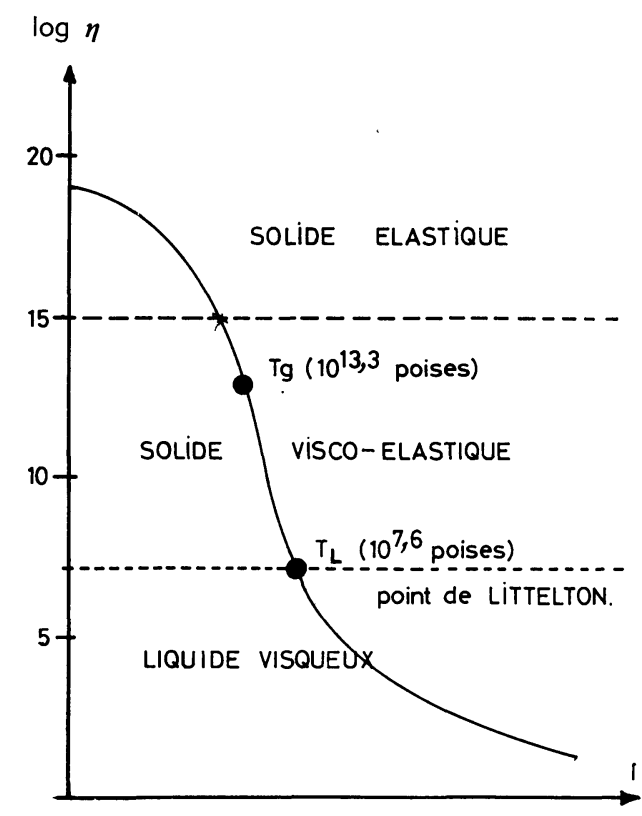

(a)

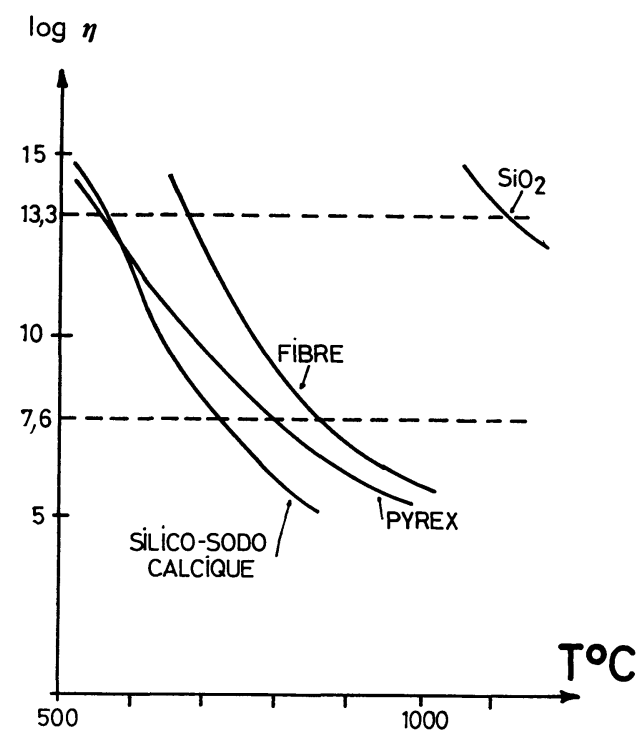

(b)

Fig. 7. - a) Allure du graphe $\log \eta=f(T)$ pour un solide vitreux. b) Graphes $\log \eta=f(T)$ pour quatre verres minéraux.
TABLEAU III

$\%$ en poids des constituants principaux de trois verres

$\begin{array}{lcccccc} & \mathrm{B}_{2} \mathrm{O}_{3} & \mathrm{SiO}_{2} & \mathrm{Na}_{2} \mathrm{O} & \mathrm{CaO} & \mathrm{Al}_{2} \mathrm{O}_{3} & \mathrm{MgO} \\ \text { Verre plat } & - & - & - & - & - & - \\ \text { Verre pyrex } & 11,5 & 80 & 13,5 & 11 & 1 & 1,5 \\ \text { Fibre de verre } & & 64 & & & 2,5 & \\ \end{array}$

Voici les valeurs approximatives de $T_{\mathrm{g}}$ et $T_{\mathrm{L}}$ pour ces quatre verres :

\begin{tabular}{lrrr} 
& $T_{\mathrm{g}}{ }^{\circ} \mathrm{C}$ & $T_{\mathrm{L}}{ }^{\circ} \mathrm{C}$ & $\Delta T$ \\
\cline { 2 - 4 } & 560 & $\overline{720}$ & $\frac{160}{}$ \\
Podocalcique & 560 & 800 & 240 \\
Fibre & 680 & 850 & 170 \\
Verre de silice & 1120 & 1580 & 460
\end{tabular}

Pour le premier et le troisième, la pente du graphe $\log \eta=f(T)$ est raide : il s'agit de verres courts difficiles à souffler au contraire du pyrex, verre long facile à souffler.

Ces faits expérimentaux s'expliquent fort bien en tenant compte de la rigidité des liaisons et de leur agencement structural.

Dans le verre silico-sodocalcique le réseau désordonné de la silice vitreuse a été adouci par un perturbateur à liaisons ioniques $\left(\mathrm{Na}_{2} \mathrm{O}\right)$ alors que dans le verre pyrex ce même réseau $\left(\mathrm{SiO}_{2}\right)$ a été adouci avec un formateur à réticulation tridimensionnelle assez lâche $\left(\mathrm{B}_{2} \mathrm{O}_{3}\right.$ dont la température de transition vitreuse à l'état pur, $T_{g}$, est de $260^{\circ} \mathrm{C}$ seulement).

Dans les deux cas on obtient ainsi une valeur de $T_{\mathrm{g}}$ assez basse $560^{\circ} \mathrm{C}$ au lieu de $1120^{\circ} \mathrm{C}$ pour la silice vitreuse.

La souplesse des liaisons ioniques du verre silicosodocalcique implique de grandes amplitudes de vibration des ions $\mathrm{Na}^{+}$, d'où une rupture partielle du réseau résiduel de la silice et une chute de la viscosité $\left(10^{-13,3} \rightarrow 10^{-7,6}\right)$ pour une augmentation modérée de la température $\left(560 \rightarrow 720^{\circ} \mathrm{C}\right)$.

Dans le verre pyrex, au contraire, l'adoucisseur est le formateur $\mathrm{B}_{2} \mathrm{O}_{3}$ à réticulation lâche mais avec des liaisons covalentes rigides qui impliquent de faibles amplitudes de vibration et il faut donc chauffer davantage $\left(560 \rightarrow 800^{\circ} \mathrm{C}\right)$ pour observer la même chute de viscosité.

Bien entendu, ce raisonnement s'applique à fortiori à la silice vitreuse qui est un verre encore plus long que le pyrex $\left(1120 \rightarrow 1580^{\circ} \mathrm{C}\right)$, mais les températures élevées auxquelles la viscosité s'abaisse rendent sa technologie de mise en forme difficile.

Dans la fibre de verre, on a adouci le réseau de la silice vitreuse avec les intermédiaires $\left(^{8}\right) \mathrm{Al}_{2} \mathrm{O}_{3}$ et $\mathrm{MgO}$ : cet adoucissement est donc plus faible que

(8) Cet adjectif précise que les cations correspondants ne sont ni des formateurs de réseau, ni des perturbateurs. 
pour le verre silico-sodocalcique et le pyrex et le graphe $\log \eta=f(T)$ se trouve donc situé entre ceux de ces deux verres et celui de la silice vitreuse : sa technologie de mise en forme nécessite donc des températures moyennes $\left(\sim 750^{\circ} \mathrm{C}\right)$.

Le verre de sélénium, Se a une transition vitreuse qui vaut $T_{\mathrm{g}}=75^{\circ} \mathrm{C}$. Cette basse température s'explique parfaitement, car dans ce verre on a des macromolécules linéaires comme dans les polymères thermoplastiques et les caoutchoucs $\left({ }^{9}\right)$.

Cette réticulation à une seule dimension correspond en fait à une coordinance de 2 le long de la macromolécule.

Ainsi pour le verre organique PMMA, plus connu sous le nom de plexiglas, on voit sur les diagrammes de traction $\sigma(\varepsilon)$ [ $\sigma=$ contrainte et $\varepsilon=$ élongation] que vers $90^{\circ} \mathrm{C}$ ce matériau perd sa rigidité et que cette température correspond approximativement à sa transition vitreuse (Fig. 8).

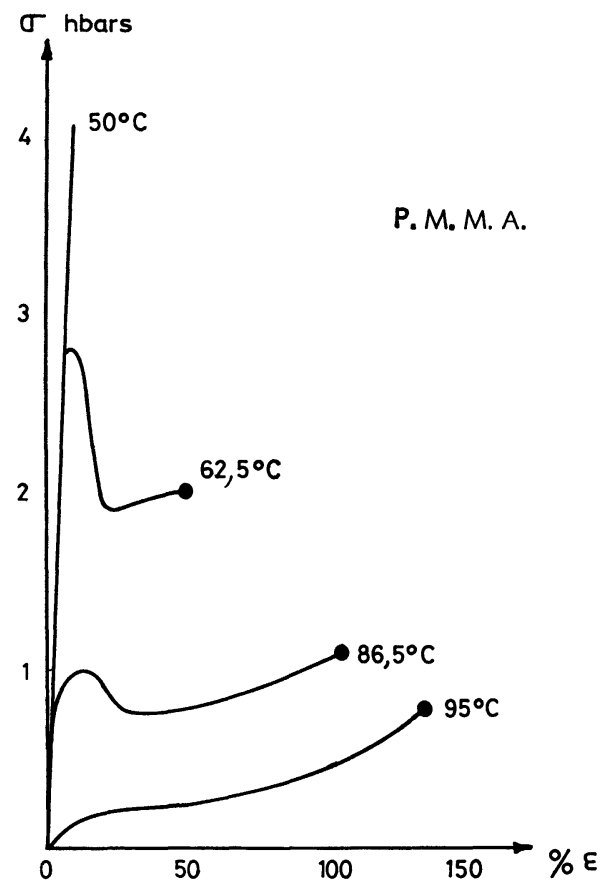

FIG. 8. - Graphes $\sigma(\varepsilon)$ à diverses températures pour le polyméthacrylate de méthyle (P. M. M. A.) avec une vitesse de traction de $100 \%$ par minute.

La transition vitreuse du polystyrène PS et des copolymères PS-POE qui sont des polymères thermoplastiques est également située vers $90^{\circ} \mathrm{C}$ (Fig. $5 b$ ) alors que celle des caoutchoucs se trouve en général vers $-70^{\circ} \mathrm{C}$.

Au contraire, les polymères thermodurcis, tels les polyesters sont réticulés à trois dimensions et on observerait une transition vitreuse à une température

(9) On néglige ici la faible réticulation tridimensionnelle qui s'établit lors de la vulcanisation du caoutchouc. beaucoup plus élevée si le polymère n'était pas détruit avant par l'agitation thermique.

Tous les exemples donnés jusqu'ici concernent des polymères amorphes : étudions maintenant les transitions thermiques d'un polymère semi-cristallin :

Le taux de cristallinité du polyéthylène basse densité est d'environ $40 \%$ si bien que ce matériau doit présenter une transition vitreuse et une fusion cristalline.

On observe effectivement sur la figure $9 a$ une transition vitreuse vers $-100^{\circ} \mathrm{C}$ et quelque chose qui ressemble à la fusion cristalline de la figure $5 a$ vers $+110^{\circ} \mathrm{C}$ mais sans en présenter la discontinuité.

Sur la figure $9 c$ l'analyse enthalpique de la fusion nous montre qu'on observe un pic de fusion très étalé

(a)
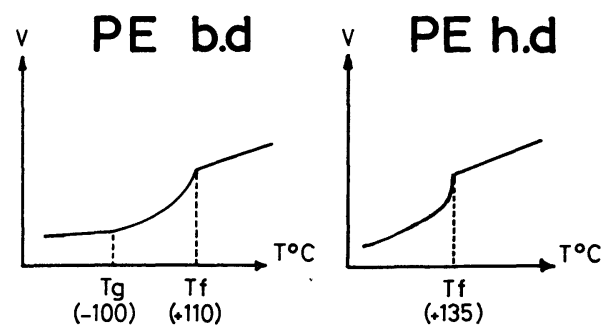

(c)

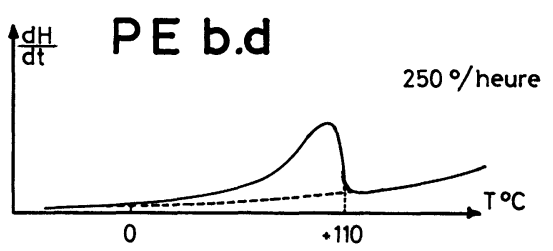

(d)

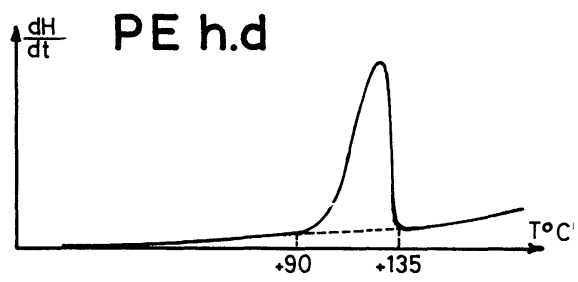

FIG. 9. $-a$ )-b) Graphes volume spécifique en fonction de la température pour les polyéthylènes haute et basse densité. c) Puissance thermique consommée lors de la fusion des polyéthylènes basse densité. $d$ ) Puissance thermique consommée lors de la fusion du polyéthylène haute densité.

entre $0^{\circ} \mathrm{C}$ (fusion commençante) et $100{ }^{\circ} \mathrm{C}$ (fusion finissante) [7]. Ceci nous prouve que les $40 \%$ de matière cristallisée contenus dans le PE basse densité correspondent à de petits agrégats très mal cristallisés.

Les résultats des figures $9 b$ et $9 d$ [7] nous prouvent que dans le PE haute densité dont le taux de cristallinité est de $85 \%$ environ les cristallites sont assez mal cristallisés. En effet on est loin d'une vraie discontinuité figure $9 b$ et d'un pic très étroit observé lors d'une fusion cristalline figure $9 d$.

Les raies de diffraction $\mathrm{X}$ élargies dont on observe les diagrammes confirment d'ailleurs ces conclusions.

5. Dévitrification, verres. Céramiques et décomposition spinodale. - On dit qu'un verre se dévitrifie 
lorsqu'il cristallise et perd ainsi les propriétés de l'état vitreux : transparence, ...

Ce phénomène arrive en particulier si on laisse longtemps le verre à une température où les deux courbes des vitesses de nucléation et de croissance se recouvrent légèrement (Fig. $6 b$ ).

Dans le cas des verres silico-sodocalciques la dévitrification se comprend à l'aide du diagramme ternaire de solidification $\mathrm{SiO}_{2}-\mathrm{Na}_{2} \mathrm{O}-\mathrm{CaO}$ (Fig. 10).

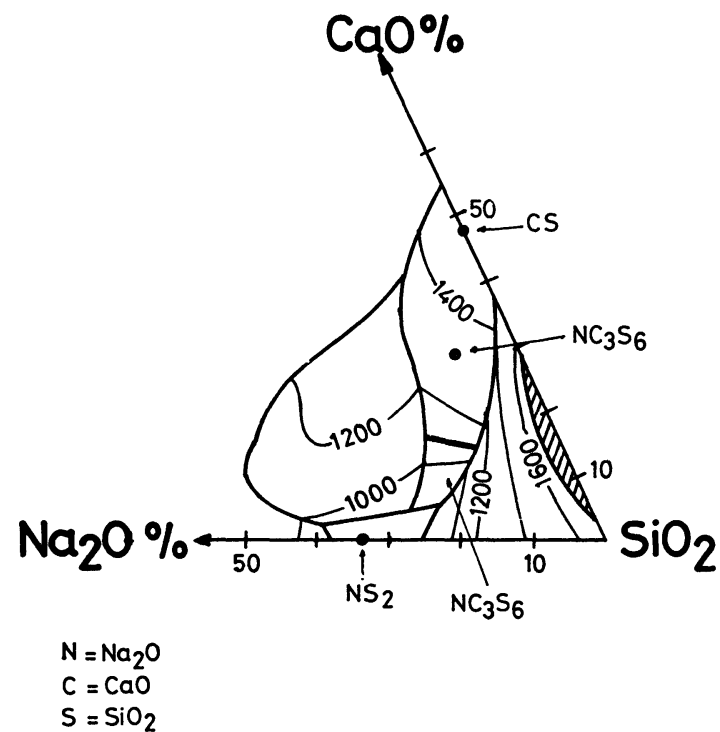

FIG. 10. - Diagramme ternaire de solidification $\mathrm{SiO}_{2}-\mathrm{Na}_{2} \mathrm{O}$ CaO. $\mathrm{N}=\mathrm{Na}_{2} \mathrm{O}, \mathrm{C}=\mathrm{CaO}, \mathrm{S}=\mathrm{SiO}_{2}$

On y observe une nappe cristobalite-tridymitequartz jouxtant la zone de démixion allongée sur l'axe $\mathrm{SiO}_{2}-\mathrm{CaO}$ (cf. Fig. 10 et Fig. $3 b$ ) descendant jusqu'à une vallée eutectique commune à cette nappe et à celles :

- du composé $\mathrm{CaO}, \mathrm{SiO}_{2}$, wollastonite, (cf. Fig. 10 et Fig. $3 b)$;

- du composé $\mathrm{Na}_{2} \mathrm{O}, 3 \mathrm{CaO}, 6 \mathrm{SiO}_{2}$, dévitrite à fusion non congruente puisque le point représentatif de ce composé ne se projette pas sur la nappe correspondante :

- du composé $\mathrm{Na}_{2} \mathrm{O}, 2 \mathrm{SiO}_{2}$ (cf. Fig. 10 et $3 a$ ).

La composition des verres silico-sodocalciques industriels se projette en général sur la nappe de la dévitrite si bien qu'on observe au microscope électronique et avec la diffraction des rayons $\mathrm{X}$ des cristaux de dévitrite lorsque ces verres se dévitrifient.

Lorsqu'on fabrique un objet en verre céramique, ou vitrocérame, on provoque la dévitrification par un traitement thermique approprié après avoir mis en forme facilement le liquide visqueux de la fonte de verre additionné d'un agent de nucléation.

L'objet en vitrocérame terminé contient en général plus de $50 \%$ de matière cristalline formée de petits cristaux de taille inférieure à $1 \mu$ et répartis dans une matrice vitreuse.
Par analogie on peut dire que les polymères semicristallins par exemple le polyéthylène, sont des vitrocérames organiques.

Dans des mélanges liquides surfondus $\mathrm{SiO}_{2}(100 \%$ à $80 \%$ en moles $) \mathrm{Na}_{2} \mathrm{O}(0 \%$ à $20 \%$ on observe une décomposition de type spinodale ou démixion subliquidus [8].

Par exemple, pour une température d'environ $750^{\circ} \mathrm{C}$ le mélange liquide surfondu $90 \% \mathrm{SiO}_{2} 10 \% \mathrm{Na}_{2} \mathrm{O}$ se

(a)

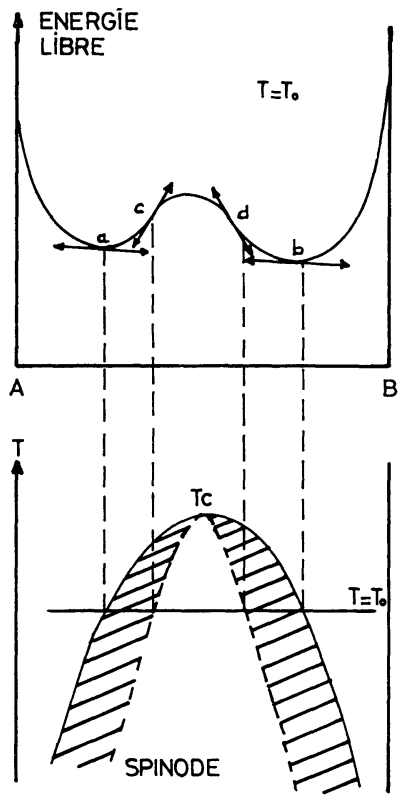

DECOMPOSITION SPINODALE

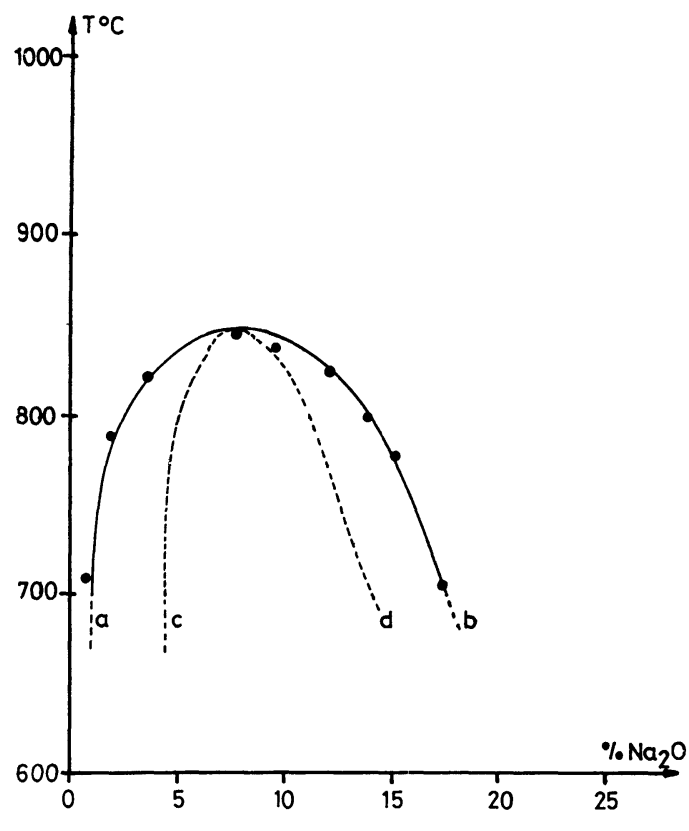

(c)

FIG. 11. - a) Graphe énergie libre en fonction de la concentration à la température $T_{0} . b$ ) Graphes température en fonction de la concentration pour les points $a, b, c$, d pour un mélange binaire général AB. $c$ ) Mêmes graphes pour le mélange binaire $\mathrm{SiO}_{2}-\mathrm{Na}_{2} \mathrm{O}$. 
sépare en deux phases : l'une d'environ $98 \%-2 \%$ et l'autre $83 \%-17 \%$.

Sur la figure $11 a$ on observe l'évolution de l'énergie libre, $G$, en fonction de la concentration, $c$, pour un mélange binaire $\mathrm{AB}$ à la température $T=T_{0}$. Entre les points d'inflexion $c$ et $d$ de ce graphe où $\partial^{2} G / \partial c^{2}=0$ la concavité est négative : $\partial^{2} G / \partial c^{2}<0$ et une petite variation de concentration entraîne donc une décroissance de la variation d'énergie libre et le système va tendre à se séparer en deux phases liquides surfondues dont la composition va évoluer vers celle des points $a$ et $b$ où l'énergie libre est minimum.

Sur la figure $11 b$ on observe les graphes concentration en fonction de la température pour les points $a, b$, c et $d$. Le lieu géométrique des points c et d s'appelle la spinode.
Sur la figure $11 c$ ces graphes ont été déterminés expérimentalement pour le mélange binaire $\mathrm{SiO}_{2}-\mathrm{Na}_{2} \mathrm{O}$.

En conclusion on a montré que la rigidité de la liaison chimique et la structure d'un solide non cristallin expliquent qualitativement ses propriétés thermodynamiques et cinétiques de changement d'état ainsi que son module d'Young macroscopique en dessous de la température de transition vitreuse.

La connaissance précise de la variation de la viscosité en fonction de la température, lors du changement continu d'état du solide non cristallin, permet de décider le meilleur procédé technologique de mise en forme du matériau correspondant. Enfin l'étude de la dévitrification d'un verre conduit à la réalisation d'objets en verre-céramique à l'aide d'un procédé de mise en forme particulièrement facile.

\section{Bibliographie}

[1] Shimanouchi, T., Tsuboi, M., Miyazawa, T., J. Chem. Phys. 35 (1961) 5. 1597.

[2] Mozzi, L., Warren, B. E., J. Appl. Cryst. 2 (1969) 164.

[3] Kozlovskaya, E. C., The structure of glass. Third All-Union Conférence on the glassy state 2 (1959).

[4] Mozzi, L., Warren, B. E., J. Appl. Cryst. 3 (1970) 59.

[5] BeCherer, G., BrÜMmer, O., HERMS, G., Röntgenographische
Strukturuntersuchungen an Na. Boratgläsern. Silikattech. 13 (1962), 339.

[6] Bray, P. J., O’Keefe, J. G., Phys. Chem. Glasses 3 (1962), 181.

[7] Espagno, L. Communication personnelle.

[8] Tomozawa, M., Mac Crone, R. K., Herman, H., Phys. Chem. Glasses 11 (1970) 136. 